\title{
A Simultaneous Multidisciplinary Evaluation of Irritable Bowel Syndrome in Egyptian Patients: Dietary, Psychiatric, Microbiologic and Autonomic Aspects
}

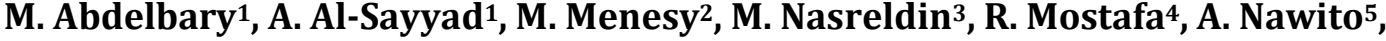 \\ R. Marzaban ${ }^{*}$ \\ ${ }^{1}$ Tropical Medicine Department, Faculty of Medicine, Cairo University, Giza, Egypt \\ ${ }^{2}$ Gastrointestinal Endoscopy Unit, Cairo, Egypt \\ ${ }^{3}$ Psychiatry Department, Faculty of Medicine, Cairo University, Giza, Egypt \\ ${ }^{4}$ Clinical and Chemical Pathology Department, Faculty of Medicine, Cairo University, Giza, Egypt \\ ${ }^{5}$ Clinical Neurophysiology Department, Faculty of Medicine, Cairo University, Giza, Egypt \\ Email: ${ }^{*}$ gymarz@yahoo.com
}

How to cite this paper: Abdelbary, M., Al-Sayyad, A., Menesy, M., Nasreldin, M., Mostafa, R., Nawito, A. and Marzaban, R. (2020) A Simultaneous Multidisciplinary Evaluation of Irritable Bowel Syndrome in Egyptian Patients: Dietary, Psychiatric, Microbiologic and Autonomic Aspects. Open Journal of Gastroenterology, 10, 214-230. https://doi.org/10.4236/ojgas.2020.108021

Received: July 20, 2020

Accepted: August 24, 2020

Published: August 27, 2020

Copyright ( 2020 by author(s) and Scientific Research Publishing Inc. This work is licensed under the Creative Commons Attribution International License (CC BY 4.0).

http://creativecommons.org/licenses/by/4.0/ (c) (i) Open Access

\begin{abstract}
Background and Aim: Irritable bowel syndrome (IBS) is a common medical disorder that may be severe enough to impair the quality of life. This study aimed to assess the role of each of dietary, psychiatric, autonomic, and microbiology background and their interactions in Egyptian patients with IBS. Patients and Methods: Forty adult patients diagnosed with IBS, equally divided into 2 groups the diarrhea predominant and the constipation predominant, were recruited from the Endoscopy Unit. Dietary assessment was done by monthly food frequency questionnaire. Psychiatric assessment was done by both the Eysenck Personality Questionnaire (EPQ) and Hopkins Symptom Checklist (HSCL-90). Microbiologic evaluation was done by faecal cultures and neurophysiologic autonomic evaluation was done via the sympathetic skin response and the parasympathetic R-R interval variation. Another 20 healthy subjects were included as control group. Results: All IBS patients were young, with significant female predominance $(\mathrm{P}=0.007)$, particularly in IBS-C group $(20 / 20 ; 100 \%, P=0.003)$. Psychologically, abnormal scores of neuroticism, extraversion and criminality, and depression, obsessive compulsion, somatization, sensitivity and anxiety in both IBS groups with particularly extraversion, criminality and depression were significantly higher in constipation subtype. Microbiologically, Bacteroids were significantly related to IBS, while Klebsiella was significantly deficient without significant difference between its groups. On the contrary, neither diet nor autonomic activity
\end{abstract}


showed any significant relation. Conclusions: IBS is a disorder induced by many factors and affected by several interacting agents, thus revealing controversial results when studied simultaneously.

\section{Keywords}

Irritable Bowel Syndrome (IBS), Eysenck Personality Questionnaire (EPQ), Hopkins Symptom Checklist (HSCL-90), Microbiota, Autonomic Activity

\section{Introduction}

Irritable bowel syndrome (IBS) is a common functional intestinal disorder constituting a major health complaint and is the most commonly diagnosed gut disease [1].

Pathogenesis of IBS is multifactorial: dietary (food sensitivity and carbohydrate malabsorption), microbiologic (altered fecal microbiota, bacterial overgrowth, and post infectious reactivity), and neurophysiologic (brain-gut interaction, altered gut motility, visceral hypersensitivity, intestinal inflammation) [2].

Patients may correlate ingestion of certain foods with their IBS complaints [1]. Dietary fermentable oligo-di-monosaccharides and polyols (FODMAP), a group that includes fructose, fructans, mannitol, lactose, xylitol, sorbitol, and galactans induces increased and prolonged hydrogen production in the intestine thus influencing the methane production, and eventually inducing local and systemic symptoms of IBS [2]. Conversely, low dietary FODMAP is effective in controlling IBS symptoms [3]. However, excessive diet restriction may lead to nutritional deficiencies. The suspected food is identified by the recurrence of symptoms once reintroduced in diet after being temporarily eliminated [4].

Anxiety, depression, and somatoform disorders are obviously associated with IBS [5] [6]. Ten percent of the varied bowel symptoms are attributed to stress [6]. The psychiatric role in the pathogenesis of IBS was proved by the significant resolution by psychologic treatment [6], and the symptomatic relief by cognitive and hypnotherapies, and dynamic psychotherapy [7].

The role of small intestinal bacterial overgrowth in the pathogenesis of IBS was proved by the resolution of IBS when eradicated by antibiotics in $48 \%$ of subjects [8]. However, the benefit of antibiotics is transient and their exact mode of action is not clear whether treating small bowel bacterial overgrowth or altering gut microbiota [4].

Functional bowel disorders are associated with autonomic disturbance [9] [10]. Heart rate variability (HRV) has considerable potential to assess the role of autonomic nervous system (ANS) fluctuations in normal healthy individuals and in patients [11], and skin sympathetic response (SSR) is a simple bed side test strongly correlated with diseases where dysautonomia was incriminated [12]. ANS assessment may be considered a new modality for clinical management of IBS [13]. 
This study aimed at evaluating the dietary, psychiatric, microbiologic and autonomic nervous activity of IBS patients.

\section{Patients and Methods}

This was a cohort simultaneous multidisciplinary evaluation of IBS. The study included patients attending the colonoscopy room at Gastrointestinal (GI) Endoscopy Unit, Kasr El-Ainy, Faculty of Medicine, Cairo University, Egypt and presented with variable lower, non-alarming GI symptoms, and with normal colonic macroscopic and microscopic examination. Colonoscopic biopsies were done to exclude any organic pathology including those with normal overlying mucosa. Upper endoscopy with duodenal biopsy was done in cases simulating IBS like Celiac disease.

\subsection{Inclusion Criteria}

- Adult patients ( $\geq 18$ years old);

- Both genders;

- Fulfilling the Rome III criteria [14] [15] i.e. at least three months of continuous or recurrent abdominal pain or discomfort that has two or more of the following features: improvement with defecation, association with a change in the frequency, and a change in stool form.

\subsection{Exclusion Criteria}

All organic colonic diseases that may simulate IBS clinical picture and any medications that may interfere with the applied tests of the test whether directly or indirectly.

- Celiac serology;

- Organic GI pathology;

- Alarming symptoms (e.g. significant weight loss, bloody diarrhea, cachexia).

- Organic neurologic affection;

- Medical condition affecting the brain-gut neurogenic pathways or any form of neuropathies; Special habits (alcoholism), Endocrinal, Cardiovascular diseases;

- Medications interfering with tests carried out.

Patients were equally divided into two groups according to their predominant motion; IBS-D and IBS-C. Twenty healthy volunteers participated in this work as control group.

All enrolled patients and control subjects were subjected to:

1) Full history taking: a) Complaint: constipation vs. diarrhea, frequency, passage of blood, mucous in stools, site of pain, distension, flatus, tenesmus, anal itching, passage of worms. b) Medical history. c) Dietary history e.g. Monthly food frequency questionnaire about common fermentable oligo-di-monosaccharides and polyols (FODMAP) foods in Egypt. It was composed of ingestion of beans, lentils/peas, refined bread, milk/dairy products, skinned vegetables/fruits, coffee, 
sweets/candies, carbonated beverages, cakes/cookies/pasta, rice, and large fatty meals. 2) Thorough clinical examination: a) General, b) Psychiatric assessment: The psychological profile of the patients was done provided that the Arabic certified translation was attached, by utilizing the following two tests. i) The Eysenck Personality Questionnaire (EPQ) [16]. It is composed of 90 statements of which subjects respond by "Yes" or "No". It has 5 scales that measure the dimensions of psychoticism, neuroticism, extraversion-introversion, lie, and criminality, provided that each one has its normal psychological range, and ii) The Hopkins Symptom Checklist (HSCL-90) scale [17]. It is a self-rating test to indicate how much he/she is distressed by each of 90 possible symptoms. This test is further scored for 9 clinical scales: somatization, obsessive-compulsive symptoms, interpersonal sensitivity, depression, anxiety, hostility, phobic anxiety, paranoid ideation, and psychoticism. Scale $>20$ is considered significantly positive. 3) Investigatory work up: a) Microbiologic assessment: Faecal microbiota were examined by repeated stool cultures to exclude infectious causes, and to identify faecal microbiota regarding type and count. Stool samples were cultured on i) a panel of selective and enriched media under aerobic and anaerobic conditions. Isolated microorganisms will be phenotypically identified by conventional biochemical testing and ii) an automated bacterial identification system; namely the VITEK ${ }^{\oplus} 2$ system (bioMérieux). Representative species of fecal bacterial flora will be cultured quantitatively, and further categorized as mild (1 $\left.\left.-999 \times 10^{5} \mathrm{CFU}\right) / \mathrm{ml}\right)$, moderate $\left(1000-99,999 \times 10^{5} \mathrm{CFU} / \mathrm{ml}\right) \& \mathrm{high}(\geq 100,000$ $\left.\times 10^{5} \mathrm{CFU} / \mathrm{ml}\right)$. b) Biochemical blood routine tests, c) Imaging i.e. Abdominal Ultrasound. d) Neurophysiologic Autonomic activity assessment: Autonomic activity is assessed by sympathetic skin response (SSR) [18], and R-R interval variation (RRIV) [11] which are noninvasive procedures to assess the autonomic nervous functions. SSR and RRIV were recorded using a Nihon Kohden Neuropack apparatus $^{\circledast}$ (Tokyo, Japan) in the Clinical Neurophysiology Unit, Kasr AlAiny Hospital. i) The SSR was recorded from the upper limb by attaching surface electrodes on the right hand with application of electrically stimuli to the left wrist. For the SSR the onset latency in seconds was calculated. ii) The RRIV was acquired at rest (RRIV) and during deep breathing (RRIV-D) [19]. The coefficient of variation (CV) was calculated to represent the RRIV. e) Statistical analysis: Data were entered on the computer using "Microsoft Office Excel Software" program (2010) for windows. Data were then transferred to the Statistical Package of Social Science Software program, version 23 (IBM SPSS Statistics for Windows, Version 23.0. Armonk, NY: IBM Corp.) to be statistically analyzed. Data were presented using range, mean, standard deviation (SD) and median for quantitative variables, and frequency and percentage for qualitative ones. Comparison between groups was performed using Kruskal Wallis test with Mann Whitney test for pairwise comparisons for quantitative variables and Chi square or Fisher's exact test for qualitative ones. Spearman correlation coefficients were calculated to assess the association between different quantitative and 
ordinal variables. $\mathrm{P}<0.05$ is considered statistically significant. In non-normally distributed data, $\mathrm{P}$ value was calculated via non parametric test. f) Ethical consideration: This study was conducted according to the declaration of Helsinki and was approved by the ethical committee of Tropical Medicine Department, Faculty of Medicine, Cairo University. A fully informative consent was signed by the IBS patients and control subjects but it was not presented for the personal data that may identify the patients.

\section{Results}

This study included 40 adult patients who attended the colonoscopy room of the Gastrointestinal Endoscopy Unit at Kasr El-Ainy Hospital, Faculty of Medicine, Cairo University. They were diagnosed to have IBS according to Rome III criteria. They were enrolled from June 2016 to January 2018 out of 335 referred colonoscopy cases (11.94\%).

The 40 IBS patients were divided into two equal groups; IBS-D and IBS-C, and 20 other healthy subjects serving as control group.

The basic personal data and complaints are shown in Table 1. All of the patients were young, insignificantly and mildly older IBS-C group, and predominantly females, particularly in IBS-C. Course of the complaint in both IBS groups was stationary. All of them were clinically free.

Dietary assessment of the studied groups is shown in Table 2. None of the questioned food types was significantly related to any of the studied groups.

The psychiatric assessment of the studied groups via EPQ scale and HSCL-90 in terms of their absolute score is shown in Table 3. All the parameters, except extraversion and lie of EPQ, were significantly higher in both IBS groups, and mostly with insignificant interdifference.

However, for many of the recorded scores lied within the normal psychologic range, the percent of patients who recorded abnormal psychiatric scores in EPQ and HSCL-90 were illustrated in Figure 1 and Figure 2 respectively to show the true and definite psychiatric trait.

Table 1. Basic personal data and complaint of the studied IBS patients.

\begin{tabular}{|c|c|c|c|c|c|c|c|c|}
\hline \multirow{2}{*}{\multicolumn{2}{|c|}{$\begin{array}{l}\text { Basic personal data } \\
\text { and complaint }\end{array}$}} & \multirow{2}{*}{$\begin{array}{l}\text { IBS-D } \\
n=20\end{array}$} & \multirow{2}{*}{$\begin{array}{l}\text { IBS-C } \\
n=20\end{array}$} & \multirow{2}{*}{$\begin{array}{l}\text { Control } \\
n=20\end{array}$} & \multicolumn{4}{|c|}{$P$ value } \\
\hline & & & & & P1 & P2 & P3 & P4 \\
\hline \multirow{2}{*}{ Personal } & Age in years & $32.9 \pm 10.1$ & $34.2 \pm 9.5$ & $32.7 \pm 7.7$ & 0.921 & 0.807 & 0.914 & 0.674 \\
\hline & Males/females & $8 / 12(40 / 60)$ & $0 / 20(0 / 100)$ & $4 / 16(20 / 80)$ & 0.007 & 0.003 & 0.301 & 0.106 \\
\hline \multirow{3}{*}{ Complaint } & & $47.1 \pm 39.1$ & $63.4 \pm 70.6$ & & & & & \\
\hline & Onset in months & $36(6-144)$ & $30(6-240)$ & & & & & \\
\hline & Pattern & $\begin{array}{c}2-4 \text { motions/day } \\
3(2-3)\end{array}$ & $\begin{array}{c}\text { Once within } 2-5 \text { days } \\
3(3-4)\end{array}$ & & & & & \\
\hline
\end{tabular}

${ }^{a}$ Quantitative parameters are expressed in mean \pm standard deviation, median (Inter quartile range), while qualitative parameters are expressed in number (percent). IBS-D = Irritable bowel disease-diarrhea, IBS-C = Irritable bowel disease-constipation. P1 = overall, P2 = IBS-D versus IBS-C, P3 = IBS-D versus control, P4 = IBS-C versus control. 
Table 2. Nutritional assessment via diet questionnaire of the studied groups.

\begin{tabular}{|c|c|c|c|c|c|c|c|}
\hline \multirow{2}{*}{$\begin{array}{l}\text { Foods of Diet } \\
\text { Questionnaire }\end{array}$} & \multicolumn{2}{|c|}{$\begin{array}{c}\text { IBS-D } \\
(\mathrm{n}=20)\end{array}$} & \multicolumn{2}{|c|}{$\begin{array}{c}\text { IBS-C } \\
(n=20)\end{array}$} & \multicolumn{2}{|c|}{$\begin{array}{l}\text { Control } \\
(n=20)\end{array}$} & \multirow{2}{*}{$\begin{array}{l}\text { P value } \\
\text { (overall) }\end{array}$} \\
\hline & $\begin{array}{c}\text { Prevalence } \\
\text { n (\%) }\end{array}$ & $\begin{array}{c}\text { Frequency } \\
\text { Mean } \pm \text { SD } \\
\text { median (IQR) }\end{array}$ & $\begin{array}{c}\text { Prevalence } \\
\text { n (\%) }\end{array}$ & $\begin{array}{c}\text { Frequency } \\
\text { Mean } \pm \text { SD } \\
\text { median (IQR) }\end{array}$ & $\begin{array}{l}\text { Prevalence } \\
\text { n (\%) }\end{array}$ & $\begin{array}{c}\text { Frequency } \\
\text { Mean } \pm S D \\
\text { median (IQR) }\end{array}$ & \\
\hline Beans & $19(95)$ & $\begin{array}{c}21 \pm 10.2 \\
25(12-30)\end{array}$ & $17(85)$ & $\begin{array}{c}19.4 \pm 12.2 \\
23(10-30)\end{array}$ & $20(100)$ & $\begin{array}{l}16.3 \pm 11.7 \\
12(6-30)\end{array}$ & 0.425 \\
\hline Lentils & $16(80)$ & $\begin{array}{c}4.8 \pm 9 \\
1.5(1-2.5)\end{array}$ & $16(80)$ & $\begin{array}{c}6.4 \pm 11.1 \\
1(1-2)\end{array}$ & $19(95)$ & $\begin{array}{l}1.4 \pm 0.8 \\
1(1-2)\end{array}$ & 0.742 \\
\hline Refined bread & $1(5)$ & $\begin{array}{c}1.5 \pm 6.7 \\
0(0)\end{array}$ & $0(0)$ & $\begin{array}{l}0 \pm 0 \\
0(0)\end{array}$ & $0(0)$ & $\begin{array}{l}0 \pm 0 \\
0(0)\end{array}$ & 0.368 \\
\hline Milk \& dairy products & $20(100)$ & $\begin{array}{l}12.8 \pm 10.8 \\
8(3.5-23)\end{array}$ & $19(95)$ & $\begin{array}{c}16.6 \pm 15.4 \\
10(5.5-30)\end{array}$ & $20(100)$ & $\begin{array}{l}15.3 \pm 10.2 \\
12(8-30)\end{array}$ & 0.528 \\
\hline Skinned vegetables & $2(10)$ & $\begin{array}{c}1.1 \pm 4.5 \\
0(0)\end{array}$ & $1(5)$ & $\begin{array}{c}0.1 \pm 0.2 \\
0(0)\end{array}$ & $3(15)$ & $\begin{array}{c}2.5 \pm 7.2 \\
0(0)\end{array}$ & 0.528 \\
\hline Skinned fruits & $0(0)$ & $\begin{array}{l}0 \pm 0 \\
0(0)\end{array}$ & $0(0)$ & $\begin{array}{l}0 \pm 0 \\
0(0)\end{array}$ & $2(10)$ & $\begin{array}{c}1.6 \pm 6.7 \\
0(0)\end{array}$ & 0.131 \\
\hline Sweets \& candies & $19(95)$ & $\begin{array}{l}4.3 \pm 4.7 \\
2(1-8)\end{array}$ & $19(95)$ & $\begin{array}{l}4.8 \pm 6.6 \\
2(1-8)\end{array}$ & $19(95)$ & $\begin{array}{c}4.7 \pm 3 \\
4(2-8)\end{array}$ & 0.470 \\
\hline Coffee & $17(85)$ & $\begin{array}{c}17.3 \pm 16 \\
14(1.5-30)\end{array}$ & $16(80)$ & $\begin{array}{l}19.8 \pm 24.8 \\
8(2.5-30)\end{array}$ & $18(90)$ & $\begin{array}{c}15.9 \pm 16.5 \\
8(3-30)\end{array}$ & 0.957 \\
\hline Carbonated drinks & $20(100)$ & $\begin{array}{c}13.1 \pm 10 \\
12(4-16)\end{array}$ & $18(90)$ & $\begin{array}{l}10.3 \pm 9.7 \\
8(2-12)\end{array}$ & $19(95)$ & $\begin{array}{l}12 \pm 10.2 \\
8(5-14)\end{array}$ & 0.523 \\
\hline Rice & $20(100)$ & $\begin{array}{c}25.7 \pm 6.3 \\
30(20-30)\end{array}$ & $20(100)$ & $\begin{array}{c}25.5 \pm 8.5 \\
30(25-30)\end{array}$ & $20(100)$ & $\begin{array}{c}19.6 \pm 10 \\
14(12-30)\end{array}$ & 0.052 \\
\hline Cake, cookies, pasta & $20(100)$ & $\begin{array}{l}19.8 \pm 16.1 \\
14(8-30)\end{array}$ & $20(100)$ & $\begin{array}{c}17.6 \pm 9.5 \\
16(10-30)\end{array}$ & $20(100)$ & $\begin{array}{c}11.5 \pm 6 \\
10(8-14)\end{array}$ & 0.081 \\
\hline Large fatty meal & $18(90)$ & $\begin{array}{c}2.7 \pm 3.8 \\
1(1-2)\end{array}$ & $14(70)$ & $\begin{array}{l}3.4 \pm 6.8 \\
1(0-3)\end{array}$ & $14(70)$ & $\begin{array}{c}2.4 \pm 6.5 \\
1(0-1.5)\end{array}$ & 0.305 \\
\hline
\end{tabular}

IBS-D = Irritable bowel syndrome diarrhea predominant, IBS-C = Irritable bowel syndrome constipation predominant, $\mathrm{SD}=\mathrm{Standard}$ deviation, $\mathrm{IQR}=$ Interquartile range. 
Table 3. Psychiatric assessment of the studied groups in terms of its absolute score.

\begin{tabular}{|c|c|c|c|c|c|c|c|c|}
\hline & \multirow{2}{*}{$\begin{array}{l}\text { Psychologic } \\
\text { assessment }\end{array}$} & \multicolumn{3}{|c|}{$\begin{array}{c}\text { Mean } \pm \text { SD } \\
\text { Median (IQR) }\end{array}$} & \multicolumn{4}{|c|}{$P$ value } \\
\hline & & $\begin{array}{l}\text { IBS-D } \\
(n=20)\end{array}$ & $\begin{array}{c}\text { IBS-C } \\
(n=20)\end{array}$ & $\begin{array}{l}\text { Control } \\
(n=20)\end{array}$ & P1 & P2 & P3 & P4 \\
\hline \multirow{5}{*}{ EPQ scale } & Psychotism & $\begin{array}{l}5.9 \pm 2.3 \\
6(4-7)\end{array}$ & $\begin{array}{l}6.1 \pm 1.8 \\
6(5-7)\end{array}$ & $\begin{array}{c}5.8 \pm 2.1 \\
5.5(4-7.5)\end{array}$ & 0.817 & 0.601 & 0.946 & 0.565 \\
\hline & Neuroticism & $\begin{array}{c}18.1 \pm 3.5 \\
19(15.5-21)\end{array}$ & $\begin{array}{c}20.4 \pm 1.1 \\
20.5(20-21)\end{array}$ & $\begin{array}{c}14.5 \pm 3.8 \\
15(12-16.5)\end{array}$ & $<0.001$ & 0.021 & 0.003 & $<0.001$ \\
\hline & Extraversion & $\begin{array}{c}7.9 \pm 5.2 \\
6.5(4-11.5)\end{array}$ & $\begin{array}{c}5.7 \pm 3.4 \\
5.5(2.5-8)\end{array}$ & $\begin{array}{c}13 \pm 4 \\
13(10-16.5)\end{array}$ & $<0.001$ & 0.222 & 0.002 & $<0.001$ \\
\hline & Lie & $\begin{array}{c}9.9 \pm 2.7 \\
9.5(8.5-12)\end{array}$ & $\begin{array}{c}11.3 \pm 2.2 \\
11(10-12.5)\end{array}$ & $\begin{array}{c}10.6 \pm 2.5 \\
10.5(9-11.5)\end{array}$ & 0.110 & 0.129 & 0.435 & 0.170 \\
\hline & Criminality & $\begin{array}{c}15.6 \pm 2.1 \\
15.5(14-16.5)\end{array}$ & $\begin{array}{c}17.1 \pm 2 \\
17(16-18)\end{array}$ & $\begin{array}{c}13 \pm 3.7 \\
11.5(10-16)\end{array}$ & 0.001 & 0.013 & 0.014 & 0.001 \\
\hline \multirow{9}{*}{ HSCL-90 } & Psychoticism & $\begin{array}{c}14.7 \pm 5.7 \\
13.5(11-18.5)\end{array}$ & $\begin{array}{c}15.9 \pm 5.1 \\
15(12.5-18)\end{array}$ & $\begin{array}{c}7.8 \pm 4 \\
7(5-9.5)\end{array}$ & $<0.001$ & 0.481 & $<0.001$ & $<0.001$ \\
\hline & Paranoia & $\begin{array}{l}9.4 \pm 2.7 \\
9(8-11)\end{array}$ & $\begin{array}{c}9.9 \pm 2 \\
10(9-11)\end{array}$ & $\begin{array}{l}6.4 \pm 2.7 \\
6(4-9)\end{array}$ & $<0.001$ & 0.206 & 0.003 & $<0.001$ \\
\hline & Phobic anxiety & $\begin{array}{l}8.4 \pm 4.1 \\
9(5-10)\end{array}$ & $\begin{array}{l}8.7 \pm 4.1 \\
8(6-12)\end{array}$ & $\begin{array}{l}3.3 \pm 2.4 \\
3(2-4)\end{array}$ & $<0.001$ & 0.664 & $<0.001$ & $<0.001$ \\
\hline & Hostility & $\begin{array}{l}6.7 \pm 3.1 \\
6(5-8)\end{array}$ & $\begin{array}{l}7.1 \pm 2.4 \\
7(5-9)\end{array}$ & $\begin{array}{l}3.9 \pm 0.8 \\
4(3-4)\end{array}$ & $<0.001$ & 0.530 & $<0.001$ & $<0.001$ \\
\hline & Anxiety & $\begin{array}{c}15.7 \pm 5.5 \\
16(11.5-18.5)\end{array}$ & $\begin{array}{c}18.2 \pm 5.8 \\
19(14.5-22)\end{array}$ & $\begin{array}{c}9.4 \pm 3.4 \\
10(8-10.5)\end{array}$ & $<0.001$ & 0.143 & $<0.001$ & $<0.001$ \\
\hline & Depression & $\begin{array}{c}27.4 \pm 7 \\
27.5(24.5-31)\end{array}$ & $\begin{array}{c}29.3 \pm 5.4 \\
29.5(28-31)\end{array}$ & $\begin{array}{c}17.7 \pm 4.2 \\
19(14-20)\end{array}$ & $<0.001$ & 0.232 & $<0.001$ & $<0.001$ \\
\hline & Interpersonal sensitivity & $\begin{array}{c}17.7 \pm 5.4 \\
20(16.5-21)\end{array}$ & $\begin{array}{c}19.5 \pm 4.4 \\
19(17-21)\end{array}$ & $\begin{array}{c}12.5 \pm 3.8 \\
13(10-16)\end{array}$ & $<0.001$ & 0.849 & 0.001 & $<0.001$ \\
\hline & Obsessive compulsive & $\begin{array}{c}19.9 \pm 3.9 \\
20(19-22)\end{array}$ & $\begin{array}{c}20.2 \pm 3.4 \\
20.5(18-22)\end{array}$ & $\begin{array}{c}13.9 \pm 3.6 \\
14.5(13-16)\end{array}$ & $<0.001$ & 0.744 & $<0.001$ & $<0.001$ \\
\hline & Somatization & $\begin{array}{c}19.7 \pm 8.4 \\
17.5(13.5-22)\end{array}$ & $\begin{array}{c}20.7 \pm 8.7 \\
18(15-25.5)\end{array}$ & $\begin{array}{c}8.1 \pm 3.5 \\
8.5(6-10.5)\end{array}$ & $<0.001$ & 0.645 & $<0.001$ & $<0.001$ \\
\hline
\end{tabular}

$\mathrm{EPQ}=$ Eysenck's personality questionnaire, HSCL-90 = Hopkins symptom checklist, IBS-D = Irritable bowel disease-diarrhea, IBS-C = Irritable bowel disease-constipation, $\mathrm{SD}=$ Standard deviation, IQR $=$ Interquartile range, $\mathrm{P} 1=$ overall, $\mathrm{P} 2=\mathrm{IBS}-\mathrm{D}$ versus IBS-C, $\mathrm{P} 3=\mathrm{IBS}-\mathrm{D}$ versus control, $\mathrm{P} 4=\mathrm{IBS}-\mathrm{C}$ versus control. 
Fecal microbiota of the studied groups by the conventional cultures and the automated VITEK test are shown in Table 4. Aspergillus was significantly high in IBS-C however fungal detection was generally very low.

The ANS assessment of the studied groups is shown in Table 5. Neither the sympathetic nor the parasympathetic showed statistically significant relation with any of the studied groups.

Correlation tests were done between every 2 of the 4 studied aspects in which IBS groups were merged together for 1) most of the previous results showed a significant difference between each of IBS groups and the control one but not between them, and 2) the rather small number of patients. Significant correlations were found in very few items. Correlation results were expressed in many and lengthy tables, thus not included in the manuscript.

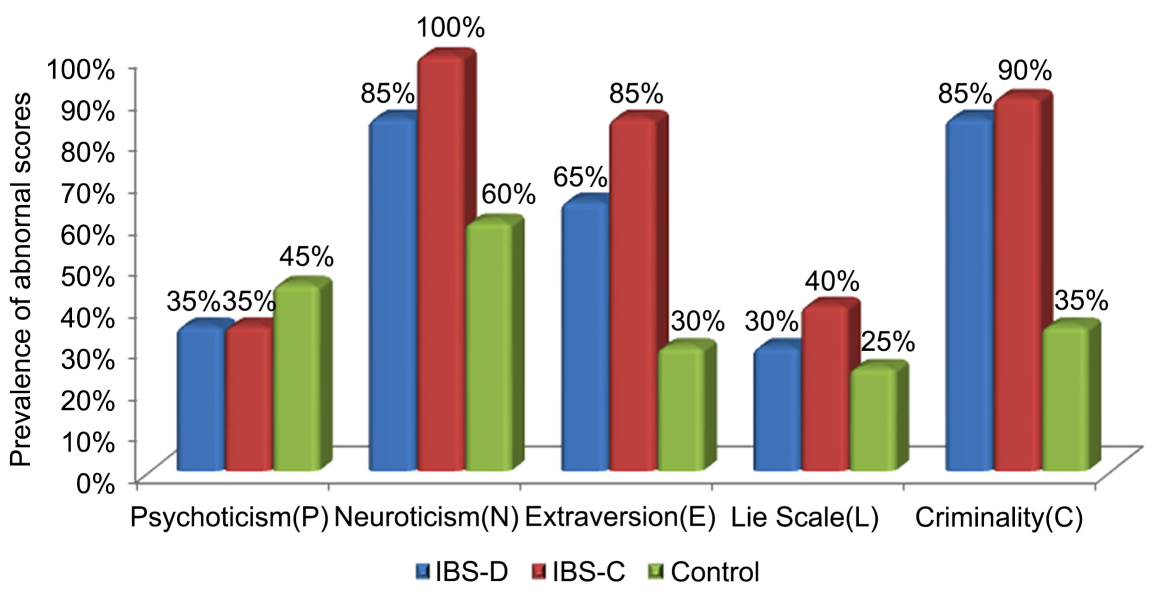

Figure 1. Prevalence of abnormal Eysneck personality questionnaire (EPQ) scores in the three studied groups $[\mathrm{P}($ overall $)=0.754,0.004,0.002,0.583$, and $<0.001$ respectively]

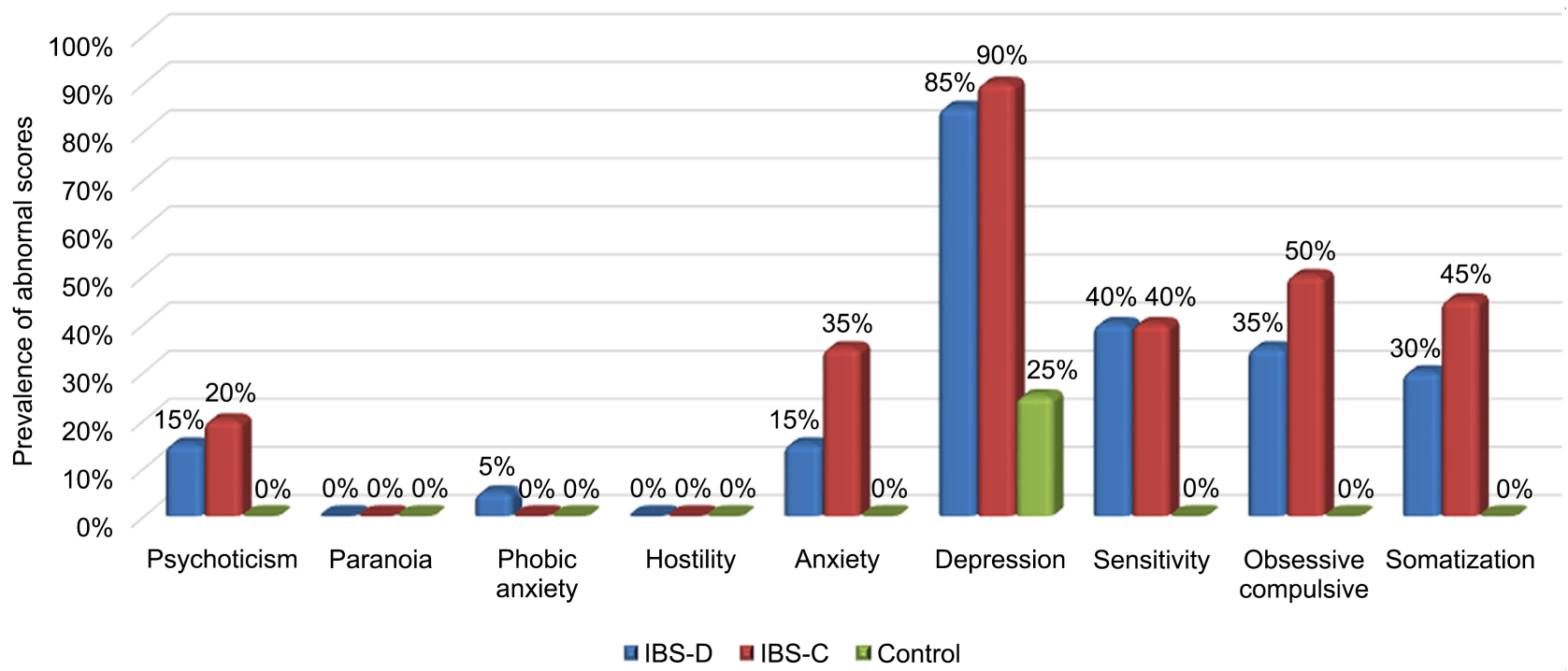

Figure 2. Prevalence of abnormal Hopkins symptom checklist (HSCL-90) scores in the three studied groups (Neither paranoia nor hostility was recorded abnormally in any of the studied groups) [P (overall) $=0.122,1.00,0.362,1.00,0.012,<0.001,0.004$, 0.002 , and 0.004 respectively]. 
Table 4. Fecal microbiota of the studied groups.

\begin{tabular}{|c|c|c|c|c|c|c|c|c|c|c|c|c|c|}
\hline \multirow{3}{*}{$\begin{array}{l}\text { Organisms' species } \\
\text { by standard culture }\end{array}$} & \multicolumn{4}{|c|}{ IBS-D $(n=20)$} & \multicolumn{4}{|c|}{ IBS-C $(n=20)$} & \multicolumn{4}{|c|}{ Controls $(\mathrm{n}=20)$} & \multirow{3}{*}{$\begin{array}{c}\text { P value } \\
\text { (Overall) }\end{array}$} \\
\hline & \multirow{2}{*}{ 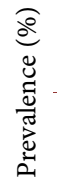 } & \multicolumn{3}{|c|}{ Degree } & \multirow{2}{*}{ 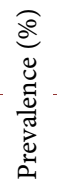 } & \multicolumn{3}{|c|}{ Degree } & \multirow{2}{*}{ 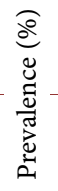 } & \multicolumn{3}{|c|}{ Degree } & \\
\hline & & Mild & Moderate & Marked & & Mild & Moderate & Marked & & Mild & Moderate & Marked & \\
\hline Coliform (enterobacterica) & 95 & 15.8 & 42.1 & 42.1 & 95 & 15.8 & 68.4 & 15.8 & 95 & 26.3 & 36.8 & 36.8 & 1.000 \\
\hline E. col & 90 & 16.7 & 38.9 & 44.4 & 95 & 26.3 & 63.2 & 10.5 & 90 & 22.2 & 44.4 & 33.3 & 0.804 \\
\hline Klebsiella & 25 & 40 & 20 & 40 & 25 & 80 & 20 & & 60 & 25 & 41.7 & 33.3 & 0.030 \\
\hline Other (non fermenters) & 10 & & 50 & 50 & 30 & 66.7 & 33.3 & & 10 & 50 & 50 & & 0.235 \\
\hline Aerobic gram positive & 80 & 25 & 56.3 & 18.8 & 95 & 31.6 & 63.2 & 5.3 & 85 & 52.9 & 35.3 & 11.8 & 0.364 \\
\hline Staphylococci & 55 & 27.3 & 72.7 & & 50 & 70 & 30 & & 55 & 54.5 & 36.4 & 9.1 & 0.176 \\
\hline Enterococci & 65 & 30.8 & 61.5 & 7.7 & 70 & 28.6 & 64.3 & 7.1 & 85 & 64.7 & 35.3 & & 0.216 \\
\hline Diphtheroid & 35 & 57.1 & 42.9 & & 30 & 83.3 & 16.7 & & 10 & 50 & 50 & & 0.155 \\
\hline Bifidobacterium & 5 & & 100 & & 20 & & 100 & & 5 & & & 100 & 0.180 \\
\hline Eubacterium & 60 & 25 & 41.7 & 33.3 & 80 & 18.8 & 50 & 31.3 & 80 & 6.3 & 43.8 & 50 & 0.256 \\
\hline Veillonella & 40 & 37.5 & 62.5 & & 50 & 20 & 70 & 10 & 65 & 61.5 & 38.5 & & 0.281 \\
\hline Peptostreptococci/Ruminococcus & 60 & 16.7 & 66.7 & 16.7 & 75 & 6.7 & 73.3 & 20 & 65 & 7.7 & 76.9 & 15.4 & 0.592 \\
\hline Fusobacterium & 35 & 28.6 & 57.1 & 14.3 & 40 & 50 & 37.5 & 12.5 & 65 & 7.7 & 76.9 & 12.5 & 0.125 \\
\hline Bacterotides & 85 & 17.6 & 52.9 & 29.4 & 90 & & 33.3 & 66.7 & 60 & 8.3 & 50 & 41.7 & 0.048 \\
\hline Prevotella & 50 & 20 & 50 & 30 & 40 & & 75 & 25 & 55 & 9.1 & 45.5 & 45.5 & 0.627 \\
\hline Clostridium & 50 & 30 & 40 & 30 & 70 & 7.1 & 57.1 & 35.7 & 65 & & 53.8 & 46.2 & 0.400 \\
\hline Actinomyces & 20 & 75 & 25 & & 5 & & 100 & & 15 & 100 & & & 0.364 \\
\hline Fungus & 10 & 50 & 50 & & 35 & 85.7 & 14.3 & & 5 & & 100 & & 0.024 \\
\hline Candida & 5 & & & & 5 & & & & & & & & \\
\hline Aspergillus & 5 & & & & 30 & & & & & & & & 0.007 \\
\hline
\end{tabular}

Organisms' species

by VITIC culture

\begin{tabular}{lccccc}
\hline & Serratia & 0 & $2(10)$ & 0 & 0.126 \\
Non fermenters & Proteus & $1(5)$ & $1(5)$ & $1(5)$ & 1.000 \\
& Pseudomonas & $1(5)$ & $3(15)$ & 0 & 0.153 \\
\hline
\end{tabular}




\begin{tabular}{|c|c|c|c|c|c|}
\hline Veillonella & Megasheria & 0 & $1(5)$ & 0 & 0.362 \\
\hline Peptostreptococci & $\begin{array}{l}\text { Peptoniphilus } \\
\text { asaccharolytica }\end{array}$ & 0 & 0 & $1(5)$ & 0.362 \\
\hline \multirow[t]{4}{*}{ Fusobacterium } & F. mortiferum & 0 & $1(5)$ & 0 & 0.362 \\
\hline & B. fragilis & $2(10)$ & $6(30)$ & $1(5)$ & 0.064 \\
\hline & B. vulgatus & 0 & $1(5)$ & 0 & 0.362 \\
\hline & B. ovatus & 0 & $3(15)$ & 0 & 0.129 \\
\hline \multirow[t]{4}{*}{ Bacteroids } & B. thetaiotamicron & 0 & $1(5)$ & 0 & 0.362 \\
\hline & B. stercoris & 0 & 0 & $1(5)$ & 0.362 \\
\hline & $\begin{array}{l}\text { Parabacteroids } \\
\text { distasonis }\end{array}$ & $1(5)$ & 0 & 0 & 0.362 \\
\hline & P. melaninogenica & $1(5)$ & $1(5)$ & $1(5)$ & 1.000 \\
\hline \multicolumn{6}{|l|}{ Prevotella } \\
\hline & P. oralis & $7(35)$ & $6(30)$ & $6(30)$ & 0.926 \\
\hline & C. sordelii & $1(5)$ & $2(10)$ & 0 & 0.349 \\
\hline & C. perfringens & $2(10)$ & 0 & 0 & 0.126 \\
\hline \multicolumn{6}{|l|}{ Clostridium } \\
\hline & C. Clostridioforme & 0 & $1(5)$ & 0 & 0.362 \\
\hline & C. sporogenes & 0 & 0 & $2(10)$ & 0.126 \\
\hline
\end{tabular}

Table 5. The autonomic activity in the three studied groups.

\begin{tabular}{|c|c|c|c|c|c|}
\hline \multirow{2}{*}{\multicolumn{2}{|c|}{ Autonomic parameters }} & \multicolumn{3}{|c|}{$\begin{array}{c}\text { Mean } \pm \text { SD } \\
\text { Median (IQR) }\end{array}$} & \multirow{2}{*}{$P$ value } \\
\hline & & $\begin{array}{l}\text { IBS-D } \\
(n=20)\end{array}$ & $\begin{array}{l}\text { IBS-C } \\
(n=20)\end{array}$ & $\begin{array}{l}\text { Control } \\
(n=20)\end{array}$ & \\
\hline \multirow{2}{*}{$\begin{array}{l}\text { Para Sympathetic } \\
\text { (coefficient) }\end{array}$} & RRIV & $\begin{array}{c}7.7 \pm 8.3 \\
4.1(2.3-10.8)\end{array}$ & $\begin{array}{c}9 \pm 10.9 \\
4.8(2.5-8.3)\end{array}$ & $\begin{array}{c}8.5 \pm 8.2 \\
4.5(3.3-10.5)\end{array}$ & 0.875 \\
\hline & RRIV-D & $\begin{array}{c}18.5 \pm 10.8 \\
19.7(12.1-27)\end{array}$ & $\begin{array}{c}28.1 \pm 24.1 \\
20.1(9.5-5.8)\end{array}$ & $\begin{array}{c}19.7 \pm 13.5 \\
15.1(11.2-26.8)\end{array}$ & 0.657 \\
\hline Sympathetic & SSR in seconds & $\begin{array}{c}2.5 \pm 1.6 \\
1.8(1.2-4.1)\end{array}$ & $\begin{array}{c}1.3 \pm 0.5 \\
1.3(1-1.5)\end{array}$ & $\begin{array}{c}1.8 \pm 1.6 \\
1.3(1-1.7)\end{array}$ & 0.079 \\
\hline
\end{tabular}

RRIV: R-R interval variation, RRIV-D: R-R interval variation during deep breathing, SSR: Sympathetic skin response. 
Beans showed the broadest (6/9 items) significant negative correlation with HSCL-90. Lentils were positively correlated with Enterococci and Actinomyces, sweets and candies were positively correlated with Klebsiella, rice was negatively correlated with Staph. aureus. Large fatty meals were positively correlated with the parasympathetic activity at both rest and deep breath tests, but neither food nor psychiatric assessment showed significant correlation with the sympathetic activity. Sympathetic activity was positively correlated with Staph. aureus, and negatively correlated with Prevotella, while the parasympathetic activity showed positive correlation with Klebsiella at rest.

\section{Discussion}

IBS is the clinical challenge in this century. It is the most commonly diagnosed GI disorder [20]. IBS is the most common complaint in gastroenterology clinics [1] [20].

In this study, the prevalence of IBS among patients referred to colonoscopy unit was $11.94 \%$. This percent was close to prevalence in several previous studies. Drossman et al., 2002 stated that almost $12 \%$ of IBS patients sought medical advice at primary care centers constituting the largest group in gastroenterology clinics [1], Talley, 1999 found that approximately $10 \%-15 \%$ of the general population have IBS [21], and Spiller et al., 2007 stated that it affects 5\% - 11\% of population of most countries [22]. It is worth mentioning that that percent in this study is like the iceberg overlying a huge number of masked patients, because the included patients are those who suffered from symptoms that were severe enough to be evaluated colonoscopically. Those with mild and moderate symptoms, besides those who do not seek medical advice were beyond our scope. Higher prevalence was reported by Meleine \& Matricon, 2014 who recorded a prevalence of one IBS patient in five people at some point in their lives, and this ratio has a significantly negative impact of both quality of life and the utility of health care centers [23].

All of the IBS patients in this study were young (mean age $=33.6 \pm 9.7$ years, IBS-D slightly younger). This agreed with Spiller et al who diagnosed IBS peak in $3^{\text {rd }}$ and $4^{\text {th }}$ decades [22].

Females were significantly predominant in IBS patients in this study $(\mathrm{P}=$ 0.007), particularly in IBS-C who were all females $(P=0.003)$, while all IBS male patients belonged to IBS-D. This was found in many previous results [21] [22]. That was explained by Meleine \& Matricon who stated that the fluctuation of ovarian hormones along the menstrual cycle affects the sensorimotor gastrointestinal function. They influence IBS onset and the pattern of its symptoms. They alter pain processing and perception by interacting with both the neuromodulatory and emotional systems. They also modulate the susceptibility and hyper-responsiveness to stress which promote immune activation or impair gut barrier function [23]. Hattori \& Fukudo, 2006 stated that females have lower pain threshold, and conversely males have greater sympathovagal balance in re- 
sponse to visceral stimulation who found $2 / 3$ of their IBS patients were females [24]. Also, Heitkemper \& Jarrett, 2008 found IBS patients seeking medical advice are mainly women for several reasons; inherent different gonadal hormones, inflammatory responses, stress reactivity, and sociocultural differences in response to pain and/or bowel pattern changes [25].

In this study none of the foods was related to IBS symptoms which were contrary to the previous results. Patients correlated foods such as caffeine, fatty foods, carbonated foods, or gas-producing foods, and alcoholic beverages as the etiology of their complaints [1]. Food plays a critical role in pathogenesis of IBS, and most patients associated its onset or exacerbation of its symptoms after certain meals. Conversely, diet low in fermentable, poorly absorbed carbohydrates and sugar alcohols is beneficial in resolving IBS symptoms [26]. In addition, increasing dietary content of soluble fiber (psyllium) significantly relieves and reduces the severity of symptoms, to the contrary the insoluble fiber (bran) worsens those symptoms [27]. Similarly, low-FODMAP diet had a favourable impact on IBS symptoms, especially abdominal pain and bloating [28]. Also, rye bread low-FODMAP helps IBS patients control their symptoms and reduces gas distension [29]. However, this finding was limited by the inability to identify the exact amount or weight of the consumed type of food by the patients, besides their similar nutritional habit.

Abnormally higher scores of neuroticism, extraversion and criminality of EPQ, and anxiety and depression of HSCL-90 were significantly recorded in IBS, where extraversion and criminality of EPQ and depression of HSCL-90 were significantly higher in IBS-C. That agreed with previous studies e.g. Chakraborti et al who proved neuroticism, hypochondriasis and depression significantly found in IBS patients compared to control subjects [29], and Zhang et al., 2018 who reported more frequent and severer depressive symptoms than healthy controls, particularly in female patients and younger age [30]. On the other hand, the findings in this study partially agreed with other studies e.g. Lee et al. 2017, and Geng et al. 2018 who stated that depression and anxiety levels are higher in IBS patients than in healthy controls, regardless IBS type [31] [32]. Anxiety and depression scores and overall symptom profiles are not significantly different in IBS subtypes. Moreover, anxiety and depression do not appear to be strongly associated with symptom severity, although they may interact with other psychological drivers of severity such as poor adapting skills [33], thus, the efficient mindfulness-based training remarkably reduces symptom severity and improves quality of life [34].

In this study, only few microbiota showed significant difference between IBS and control subjects. Bacteriodes were significantly high while Klebsiella was significantly low in both IBS groups. On the other hand, Aspergillus was significantly high in IBS-C group; however, fungal detection was generally low. Role of intestinal dysbiosis was proved in many studies but with different and even contradictory results [35] e.g. Jeffery et al., 2012 reported significant decrease of 
Bacteroids in IBS [36], Si et al., 2004 found significant increase and decrease of Enterobacteriaceae and Bifidobacterium respectively in IBS patients compared with healthy controls [37], Kennedy et al., 2014 found significantly lower Bifidobacteria and Lactobacilli [36], and Mättö et al., 2005 found slightly higher numbers of coliforms as well as an increased aerobe: anaerobe ratio in IBS [38]. Also, Bifidobacteria were higher in IBS-C yet insignificantly. As a probiotic, Bifidobacterium infantis 35624 efficiently improved IBS symptoms like bloating/distention, abdominal pain/discomfort, and/or bowel movement [39]. However, probiotics showed controversial results in pathogenesis and treatment of IBS that need to be further clarified [40].

Neither the sympathetic nor the parasympathetic activities, the two components of the ANS, were significantly related to IBS. This agreed with several studies [41] [42] [43] [44] [45], for the applied autonomic activity tests in this study do not reflect the gastrointestinal vagal tone [46]. To the contrary, other studies proved the role of ANS dysfunction in the pathophysiology of IBS [13] [47] [48].

In this study, correlative tests were done between each two studied aspects including all their items. Both diet and faecal micobiota showed few significant intercorrelation and with psychiatric and autonomic activity parameters as well. The diet provides elements for microbial fermentation, and consequently triggering IBS symptomatology. In addition, definite interaction between certain foods and the intestinal microbiota is likely one of the explanations for the inconclusive findings among studies comparing the microbiota composition in IBS patients and healthy subjects [49]. Thus, Harper et al. 2018 recommended combined therapy of probiotics and low FODMAP diet as the ideal treatment strategy for IBS [50].

Only psychiatric-autonomic correlation study was completely insignificant. That was contrary to Salvioli et al., 2015 who confirmed that correlation [13].

\section{Conclusion}

In this study, psychiatric assessment was the major parameter influencing the pathogenesis of IBS, with incorporation of few micobiota.

\section{Limitation of the Study}

The small number of the included patients for it took us carrying out 335 colonoscopies within one and half year to enroll the designed 20 IBS-D and 20 IBS-C patients to be subjected to the four parameters simultaneously. Also, the applied food frequency questionnaire did not cover all types of foods or fluids nor specify the exact quantity of the ingested type.

\section{Disclosure Statement}

All the authors declare that this study is not published or submitted for publication in any other journal. They all approved this manuscript. They also declare 
that it is unfunded with no conflict of interest whether personal or financial.

\section{Conflicts of Interest}

The authors declare no conflicts of interest regarding the publication of this paper.

\section{References}

[1] Drossman, D., Camilleri, M., Mayer, E. and Whitehead, W. (2002) AGA Technical Review on Irritable Bowel Syndrome. Gastroenterology, 123, 2108-2131.

https://doi.org/10.1053/gast.2002.37095

[2] Ong, D.K, Mitchell, S.B., Barrett, J.S., Shepherd, S.J., Irving, P.M., Biesiekierski, J.R., Smith, S., Gibson, P.R. and Muir, J.G. (2010) Manipulation of Dietary Short Chain Carbohydrates Alters the Pattern of Gas Production and Genesis of Symptoms in Irritable Bowel Syndrome. Journal of Gastroenterology and Hepatology, 25, 1366-1373. https://doi.org/10.1111/j.1440-1746.2010.06370.x

[3] Staudacher, H.M., Whelan, K., Irving, P.M. and Lomer, M.C.E. (2011) Comparison of Symptom Response Following Advice for a Diet Low in Fermentable Carbohydrates (FODMAPs) versus Standard Dietary Advice in Patients with Irritable Bowel Syndrome. Journal of Human Nutrition and Dietetics, 24, 487-495. https://doi.org/10.1111/j.1365-277X.2011.01162.x

[4] Occhipinti, K. and Smith, J.W. (2012) Irritable Bowel Syndrome: A Review and Update. Clinics in Colon and Rectal Surgery, 25, 46-52. https://doi.org/10.1055/s-0032-1301759

[5] Drossman, D.A, Creed, F.H., Olden, K.W., Svedlund, J., Toner, B.B. and Whitehead, W.E. (1999) Psychoscial Aspects of the Functional Gastrointestinal Disorders. Gut, 45, 25-30. https://doi.org/10.1136/gut.45.2008.ii25

[6] Whitehead, W.E. and Crowell, M.D. (1991) Psychologic Considerations in the Irritable Bowel Syndrome. Gastroenterology Clinics of North America, 20, 249-67.

[7] Brandt, L.J., Chey, W.D., Foxx-Orenstein, A.E., Schiller, L.R., Schoenfeld, P.S., Spiegel, B.M., Talley, N.J. and Quigley, E.M.M. (2009) American College of Gastroenterology Task Force on Irritable Bowel Syndrome. An Evidence-Based Position Statement on the Management of Irritable Bowel Syndrome. The American Journal of Gastroenterology, 104, S8-S35.

https://doi.org/10.14309/00000434-200901001-00002

[8] Pimentel, M., Chow, E.J. and Lin, H.C. (2000) Eradication of Small Intestinal Bacterial Overgrowth Reduces Symptoms of Irritable Bowel Syndrome. The American Journal of Gastroenterology, 95, 3503-3506.

[9] Bharucha, A.E., Camilleri, M., Low, P.A. and Zinsmeister, A.R. (1993) Autonomic Dysfunction in Gastrointestinal Motility Disorders. Gut, 34, 397-401.

https://doi.org/10.1136/gut.34.3.397

[10] Tougas, G. (2000) The Autonomic Nervous System in Functional Bowel Disorders. Gut, 47, iv78-80. https://doi.org/10.1136/gut.47.suppl_4.iv78

[11] Malik, M. (1996) Heart Rate Variability: Standards of Measurement, Physiological Interpretation and Clinical Use. Circulation, 93, 1043-1065.

[12] Arunodaya, G.R. and Taly, A.B. (1995) Sympathetic Skin Response: A Decade Later. Journal of the Neurological Sciences, 129, 81-89. https://doi.org/10.1016/0022-510X(94)00265-P 
[13] Salvioli, B., Pellegatta, G., Malacarne, M., Pace, F., Malesci, A., Pagani, M. and Lucini, D. (2015) Autonomic Nervous System Dysregulation in Irritable Bowel Syndrome. Journal of Neurogastroenterology and Motility, 27, 423-430. https://doi.org/10.1111/nmo.12512

[14] Drossman, D.A. (2006) The Functional Gastrointestinal Disorders and the Rome III Process. Gastroenterology, 130, 1377-1390. https://doi.org/10.1053/j.gastro.2006.03.008

[15] Longstreth, G.F., Thompson, W.G., Chey, W.D., Houghton, L.A., Mearin, F. and Spiller, R.C. (2006) Functional Bowel Disorders. Gastroenterology, 130, 1480-1491. https://doi.org/10.1053/j.gastro.2005.11.061

[16] Eysenck, H.J and Eysenck, S.B.G. (1975) Manual of the Eysenck Personality Questionnaire. Hodder and Stoughton, London. https://doi.org/10.1037/t05462-000

[17] Derogatis, L., Lipman, R., Reckles, K., Uhlenhuth, E. and Covi, L. (1974) Hopkins Symptom Checklist (HSL). Self-Report Symptom Inventory. Behavioral Science, 19, 1-15. https://doi.org/10.1002/bs.3830190102

[18] Claus, D. and Schondorf, R. (1999) Sympathetic Skin Response. Electroencephalography and Clinical Neurophysiology, 52, 277-282.

[19] Ewing, D.J., Martyn, C.N., Young, R.J. and Clarke, B.F. (1985) The Value of Cardiovascular Autonomic Function Tests: 10 Years Experience in Diabetes. Diabetes Care, 8, 491-498. https://doi.org/10.2337/diacare.8.5.491

[20] Soares, R. (2014) Irritable Bowel Syndrome: A Clinical Review. World Journal of Gastroenterology, 20, 12144-12160. https://doi.org/10.3748/wjg.v20.i34.12144

[21] Talley, N.J. (1999) Irritable Bowel Syndrome: Definition, Diagnosis and Epidemiology. Best Practice \& Research Clinical Gastroenterology, 13, 371-384. https://doi.org/10.1053/bega.1999.0033

[22] Spiller, R., Aziz, Q., Creed, F., Emmanuel, A., Houghton, L., Hungin, P., Jones, R., Kumar, D., Rubin, G., Trudgill, N. and Worwell, P. (2007) Guidelines on the Irritable Bowel Syndrome: Mechanisms and Practical Mangament. Gut, 56, 1770-1798. https://doi.org/10.1136/gut.2007.119446

[23] Meleine, M. and Matricon, J. (2014) Gender-Related Differences in Irritable Bowel Syndrome: Potential Mechanisms of Sex Hormones. World Journal Gastroenterology, 20, 6725-6743. https://doi.org/10.3748/wjg.v20.i22.6725

[24] Hattori, T. and Fukudo, S. (2006) Effects of Gender on Irritable Bowel Syndrome. Nihon Rinsho, 64, 1549-1551.

[25] Heitkemper, M. and Jarrett, M. (2008) Irritable Bowel Syndrome: Does Gender Matter? Journal of Psychosomatic Research, 64, 583-587. https://doi.org/10.1016/j.jpsychores.2008.02.020

[26] Cuomo, R., Andreozzi, P., Zito, F.P., Passananti, V., De Carlo, G. and Sarnelli, G. (2014) Irritable Bowel Syndrome and Food Interaction. World Journal of Gastroenterology, 20, 8837-8845.

[27] Bijkerk, C.J., de Wit, N.J., Muris, J.W.M., Whorwell, P.J., Knottnerus, J.A. and Hoes, A.W. (2009) Soluble or Insoluble Fibre in Irritable Bowel Syndrome in Primary Care? Randomised Placebo Controlled Trial. BMJ, 339, b3154. https://doi.org/10.1136/bmj.b3154

[28] Altobelli, E., Del Negro, V., Angeletti, P. and Latella, G. (2017) Low-FODMAP Diet Improves Irritable Bowel Syndrome Symptoms: A Meta-Analysis. Nutrients, 9, 940. https://doi.org/10.3390/nu9090940

[29] Chakraborti, S.K., Dey, B.K., Ghosh, N., Chaudhury, A.N. and Guha Mazumder, 
D.N. (1996) Objective Evaluation of Psychological Abnormality in Irritable Bowel Syndrome. Indian Journal of Gastroenterology, 15, 43-45.

[30] Zhang, Q.-E., Wang, F., Geng. Q., Zheng, W., Ng, C.H., Ungvari, G.S., Yuan, Z., Mei, S., Wang, G. and Xiang, Y.-T. (2018) Depressive Symptoms in Patients with Irritable Bowel Syndrome: A Meta-Analysis of Comparative Studies. International Journal of Biological Sciences, 14, 1504-1512. https://doi.org/10.7150/ijbs.25001

[31] Lee, C., Doo, E., Choi, J.M., Jang, S., Ryu, H.-S., Lee, J.Y., Oh, J.H., Park, J.H. and Kim, Y.S., Brain-Gut Axis Research Group of Korean Society of Neurogastroenterology and Motility (2017) The Increased Level of Depression and Anxiety in Irritable Bowel Syndrome Patients Compared with Healthy Controls: Systematic Review and Meta-analysis. Journal of Neurogastroenterology and Motility, 23, 349-362. https://doi.org/10.5056/jnm16220

[32] Geng, Q., Zhang, Q.-E., Wang, F., Zheng, W., Ng, C.H., Ungvari, G.S., Wang, G. and Xiang, Y.-T. (2018) Comparison of Comorbid Depression between Irritable Bowel Syndrome and Inflammatory Bowel Disease: A Meta-Analysis of Comparative Studies. Journal of Affective Disorders, 237, 37-46. https://doi.org/10.1016/j.jad.2018.04.111

[33] de Castro, N.G.R., Miller, V., Carruthers, H.R. and Whorwell, P.J. (2015) Irritable Bowel Syndrome: A Comparison of Subtypes. Journal of Gastroenterology and Hepatology, 30, 279-285. https://doi.org/10.1111/jgh.12704

[34] Gaylord, S.A., Palsson, O.S., Garland, E.L., Faurot, K.R., Coble, R.S., Mann, J.D., Frey, W., Leniek. K. and Whitehead, W.E. (2011) Mindfulness Training Reduces the Severity of Irritable Bowel Syndrome in Women: Results of a Randomized Controlled Trial. The American Journal of Gastroenterology, 106, 1678-1688.

https://doi.org/10.1038/ajg.2011.184

[35] Rodiño-Janeiro, B.K., Vicario, M., Alonso-Cotoner, C., Pascua-García, R. and Santos, J. (2018) A Review of Microbiota and Irritable Bowel Syndrome: Future in Therapies. Advances in Therapy, 35, 289-310. https://doi.org/10.1007/s12325-018-0673-5

[36] Kennedy, P.J., Cryan, J.F., Dinan, T.G. and Clarke, G. (2014) Irritable Bowel Syndrome: A Microbiome-Gut-Brain Axis Disorder? World Journal of Gastroenterology, 20, 14105-14125. https://doi.org/10.3748/wjg.v20.i39.14105

[37] Si, J.-M., Yu, Y.-C., Fan, Y.-J. and Chen, S.-J. (2004) Intestinal Microecology and Quality of Life in Irritable Bowel Syndrome Patients. World Journal of Gastroenterology, 10, 1802-1805. https://doi.org/10.3748/wjg.v10.i12.1802

[38] Mättö, J., Maunuksela, L., Kajander, K., Palva, A., Korpela, R., Kassinen, A. and Saarela, M. (2005) Composition and Temporal Stability of Gastrointestinal Microbiota in Irritable Bowel Syndrome-A Longitudinal Study in IBS and Control Subjects. FEMS Immunology and Medical Microbiology, 43, 213-222. https://doi.org/10.1016/j.femsim.2004.08.009

[39] Brenner, D.M., Moeller, M.J., Chey, W.D. and Schoenfeld, P.S. (2009) The Utility of Probiotics in the Treatment of Irritable Bowel Syndrome: A Systematic Review. The American Journal of Gastroenterology, 104, 1033-1049.

[40] Lee, B.J., Bak and Y.-T. (2011) Irritable Bowel Syndrome, Gut Microbiota and Probiotics. Journal of Neurogastroenterology and Motility, 17, 252-266. https://doi.org/10.5056/jnm.2011.17.3.252

[41] Jarrett, M.E., Burr, R.L., Cain, K.C., Rothermel, J.D., Landis, C.A. and Heitkemper, M.M. (2008) Autonomic Nervous System Function During Sleep Among Women with Irritable Bowel Syndrome. Digestive Diseases and Sciences, 53, 694-703. https://doi.org/10.1007/s10620-007-9943-9 
[42] Ng, C., Malcolm, A., Hansen, R. and Kellow, J. (2007) Feeding and Colonic Distension Provoke Altered Autonomic Responses in Irritable Bowel Syndrome. Scandinavian Journal of Gastroenterology, 42, 441-446. https://doi.org/10.1080/00365520600965749

[43] Robert, J.J.T., Orr, W.C. and Elsenbruch, S. (2004) Modulation of Sleep Quality and Autonomic Functioning by Symptoms of Depression in Women with Irritable Bowel Syndrome. Digestive Diseases and Sciences, 49, 1250-1258. https://doi.org/10.1023/B:DDAS.0000037820.54069.6c

[44] Heitkemper, M., Jarrett, M., Cain, K.C., Burr, R., Levy, R., Feld, A. and Hertig, V. (2001) Autonomic Nervous System Function in Women with Irritable Bowel Syndrome. Digestive Diseases and Sciences, 46, 1276-1284. https://doi.org/10.1023/A:1010671514618

[45] Elsenbruch, S., Lovallo, W.R. and Orr, W.C. (2001) Psychological and Physiological Responses to Postprandial Mental Stress in Women with the Irritable Bowel Syndrome. Psychosomatic Medicine, 63, 805-813. https://doi.org/10.1097/00006842-200109000-00014

[46] Park H-J. (2008). Heart Rate Variability as a Measure of Disease State in Irritable Bowel Syndrome. Asian Nursing Research, 2, 5-16. https://doi.org/10.1016/S1976-1317(08)60024-9

[47] Dobrek, L., Friediger, J., Furgała, A. and Thor, P.J. (2006) Autonomic Nervous System Activity in IBS Patients Estimated by Heart Rate Variability (HRV). Przeglagd Lekarski, 63, 743-747.

[48] Mazurak, N., Seredyuk, N., Sauer, H., Teufel, M. and Enck, P. (2012) Heart Rate Variability in the Irritable Bowel Syndrome: A Review of the Literature. Neurogastroenterology and Motility, 24, 206-216. https://doi.org/10.1111/j.1365-2982.2011.01866.x

[49] Rajilić-Stojanović, M., Jonkers, D.M., Salonen, A., Hanevik, K., Raes, J., Jalanka, J., de Vos, W.M., Manichanh, C., Golic, N., Enck, P., Philippou, E., Iraqi, F.A., Clarke, G., Spiller, R.C. and Penders, J. (2015) Intestinal Microbiota And Diet in IBS: Causes, Consequences, or Epiphenomena? The American Journal of Gastroenterology, 110, 278-287. https://doi.org/10.1038/ajg.2014.427

[50] Harper, A., Naghibi, M. and Garcha D. (2018) The Role of Bacteria, Probiotics and Diet in Irritable Bowel Syndrome. Foods, 7, 13.

https://doi.org/10.3390/foods7020013 